\title{
Evolution of growth density equation by constraints on effective Newtonian constant $G_{e f f}$
}

\author{
Abraão J.S. Capistrano ${ }^{1,2 *}$
}

The acceleration of the universe is described as a consequence of the extrinsic curvature of the space-time embedded in a bulk space, defined by the EinsteinHilbert. Using the linear approximation of Nash-Green theorem, we obtain the related perturbed equations in which just the gravitational-tensor field equations contribute to propagation of cosmological perturbations. In accordance with Big Bang Nucleosynthesis and solar constraints, we calculate numerically the effective Newtonian function $G_{\text {eff }}$ to constrain the related parameters of the model. We numerically solve the growth density equation for two possible family of solutions leading to an interesting overdensity and, in some cases, a mild damping of the growth profiles, with a top amplification of the growth perturbations around $14 \%$ in comparison with $\Lambda$ CDM model and quintessence. The effective gravitational $\Phi$ and Newtonian curvature $\Psi$ are also analysed showing mild perturbations in early times induced only by the extrinsic curvature differently from the $\Lambda$ CDM standards.

\section{Introduction}

In the last decades, the dark energy problem turns one of the central debates on the contemporary physics. Following Occam's razor principle, the simplest explanation for the observed accelerated expansion of the universe lies within the $\Lambda$ CDM paradigm that invariably leads to the concept of dark energy: an exotic fluid with negative pressure that drives the universe to speed up. The paradigm reveals still unanswered questions about the underlying physics or in the lack of a fundamental principle or a definite observational evidence that justifies/proves the existence of the Cold dark matter and the relevance of the cosmological constant $\Lambda$. The nature of the cosmological constant is also a problem of its own with the coincidence problems (on the reason of why the matter distribution

\footnotetext{
* Corresponding author E-mail: abecapistrano@gmail.com

1 Federal University of Latin-American Integration, Foz do Iguassu-PR, 85867-670, Brazil
}

and $\Lambda$-dark energy content are seen today as the same order of magnitude) and fine-tuning issues. From the semi-classical Einstein's equations, quantum field theory suggests that the vacuum states of quantum fields contribute to a non-zero, constant energy density $\rho_{v a c}$, which produces an on going perturbation of the gravitational field in cosmology or, equivalently, in the space-time metric. References on these problems can be found in [1-7]. Notwithstanding the $\Lambda \mathrm{CDM}$ model responds positively to a vast observational evidences of accelerating expansion of the universe [8-20], it does not explain the observed dwarf galaxy populations in the Local group [21-23] and the CDM simulations lead to a discrepancy of the size of a dark matter halo core in comparison with the observed scale length of the galactic disk due to sub-galactic central cusp structures [24-28]. For a review at small scale problems within the $\Lambda$ CDM paradigm, see Ref. [29]. We also add the 2- $\sigma$ tension in the $\sigma_{8}$-contours of growth of r.m.s fluctuations on the scale of $8 h^{-1} \mathrm{Mpc}$ with a mismatch of the data inferred from Planck Cosmic Microwave Background (CMB) radiation and Large Scale Structure (LSS) observations within $\Lambda$ CDM background, which might be a result from systematics or due to deviations of gravity [30]. The problem persists even if one does not consider the Planck CMB data [31] and other evidences indicate similar tensions in the matter distributions around 2- $\sigma$ [20,32-34].

The lack of robust solutions from the aforementioned problems led to alternative models to the standard $\Lambda \mathrm{CDM}$, for instance, the dark energy models [35-37], the modified gravity models that change the standard General Relativity (GR) such as F(R) and variants [38-47] and references therein. In a different direction, we have investigated the embedding of geometries and their consequences to a physical theory in a different approach as compared with extra-dimensions models on superstrings 
or M theories inspired, such as Randall-Sundrum braneworld cosmology and variants [48-50]. In particular, the Randall-sundrum model uses the algebraic Israel- Lanczos boundary condition as a relation between the extrinsic curvature and the material sources. The theoretical consequence is that the application of such condition does not follow the evolution of the embedded geometry [51]. In this paper, apart from the braneworld context, we study the consequences when considering the universe as an embedded space-time dynamically evolving by perturbations of the Nash-Greene embedding theorem [52, 53]. Based on the model proposed in [54-57], we tested the model at background level in a series of previous works [58-62]. Specifically it passed through the cosmokinetics diagnosis, as shown in Ref. [58,61]. We follow this direction going further on the context of cosmological perturbations. In this sense, we intend to propose a relevance of a renewed concept of Riemmanian curvature with the addition of the extrinsic curvature and the Nash-Greene embedding theorem as an oriented mathematical ground.

The outline of paper is as follows. In the section 2 we present a summary of the embedding process presenting the field equations. Using the linear approximation of Nash-Green theorem, it is shown the background cosmological equations in compliance with erstwhile published results $[57,58,61]$. We also investigate the Friedmann equation modified by extrinsic curvature that detains two possible signature $\Gamma^{ \pm}$-solutions and we study a family of these solutions for allowed values of the parameters. In the section 3 , we obtain the perturbed equations in a conformal Newtonian frame and the related growth of contrast matter. To solve the growth equation, we numerically study the effective Newtonian Gravitational constant $G_{\text {eff }}$ to obtain a constraint on the parameters. Hence, a numerical analysis is made on the growth equations in comparison with $\Lambda \mathrm{CDM}$ model and GR quintessence. The effective gravitational $\Phi$ and Newtonian curvature $\Psi$ are also analysed. Final remarks are made in the conclusion section.

\section{The universe as an embedded space}

In this paper we use the same theoretical background of a series of previous works [54-62] in which is possible the description of dynamical embedding without any junction conditions unlike that of rigid embedding models $[48,49]$. A common bottom line between braneworld models and other embedding models (this works is an example) is the embedding itself and how to define the dynamics of extrinsic curvature once the embedding can be made in several different forms (e.g., local or global, isometric, analytic or differentiable etc.). It is important to point out that the junction conditions are not unique [63-67] leading to different physical results [68] and they can be completely ignored depending on the related embedding framework such as the seminal works of ArkaniHamed, Dimopoulos and Dvali, commonly known as ADD model [69] or the DGP model, as an acronym for Dvali, Gabadadze and Porrati's model [50]. In this work, we use the Nash-Greene differentiable embedding $[52,53]$ as a principle for dynamical embedding.

\subsection{The Nash-Greene embedding process}

The main result of the Nash-Greene embedding theorem $[52,53]$ is applicable to an arbitrary number of dimensions. It shows that the evolution of any embedded $n$-dimensional Riemannian geometry $V_{n}$ in an Ndimensional larger geometry (bulk) is possible by a sequence of infinitesimal perturbations of a non-perturbed background metric $\tilde{g}_{\mu \nu}$ given by the non-perturbed extrinsic curvature tensor $\tilde{k}_{\mu v a}$. In the case of interest, in a five dimensional bulk $V_{5}$, the embedding of a four dimensional space can be given by the map $\mathscr{X}: V_{4} \rightarrow V_{5}$. The fluctuations of $V_{4}$ lead to a new (perturbed) geometry $g_{\mu v}$

$$
g_{\mu v}=\tilde{g}_{\mu v}+\delta y \tilde{k}_{\mu v}+(\delta y)^{2} g^{\rho \sigma} \tilde{k}_{\mu \rho} \tilde{k}_{v \sigma} \cdots
$$

and one can obtain linear Nash fluctuations as

$\delta g_{\mu v}=-2 \tilde{k}_{\mu \nu} \delta y$,

where $\delta y$ denotes an infinitesimal displacement of the extra dimension $y$ in the bulk space and it is not considered in the line elements as in the case of rigid embedding models $[48,49]$. A similar expression of Eq.(1) was obtained year latter after Nash's seminal work in the ADM quantization program [55]. Since the extension to PseudoRiemannian manifolds was made by Greene [53], hereon we refer as Nash-Green theorem. Concerning notation, we adopt the Landau time-like convention $(---+)$ for the signature of the four dimensional embedded metric and speed of light $c=1$. Capital Latin indices run from 1 to $n$. Small case Latin indices refer to the only one extra dimension considered. All Greek indices refer to the embedded space-time counting from 1 to 4 . Non-perturbed quantities are denoted by the tilde symbol.

One can define the action $S$ as

$S=-\frac{1}{2 \kappa_{5}^{2}} \int \sqrt{|\mathscr{G}|} \mathscr{R}^{5} d^{5} x-\int \sqrt{|\mathscr{G}|} \mathscr{L}_{m}^{*} d^{5} x$,

where $\kappa_{5}^{2}$ is the energy scale on the embedded space, ${ }^{5} \mathscr{R}$ denotes the five dimensional Ricci scalar of the bulk and 
$\mathscr{L}_{m}^{*}$ denotes the confined matter Lagrangian. Thus, the variation of Einstein-Hilbert action in Eq.(2) with respect to the bulk metric $\mathscr{G}_{A B}$ leads to the Einstein equations for the bulk

${ }^{5} \mathscr{R}_{A B}-\frac{1}{2} \mathscr{G}_{A B}=\alpha^{\star} \mathscr{T}_{A B}$,

where $\alpha^{\star}$ is the energy scale parameter and $\mathscr{T}_{A B}$ is the energy-momentum tensor for the bulk [54-57].

This model can be regarded as a four-dimensional hypersurface that evolves in a five-dimensional bulk with constant curvature whose related Riemann tensor is

$$
{ }^{5} \mathscr{R}_{A B C D}=K_{*}\left(\mathscr{G}_{A C} \mathscr{G}_{B D}-\mathscr{G}_{A D} \mathscr{G}_{B C}\right), \quad A . . D=1 \cdots 5,
$$

where $\mathscr{G}_{A B}$ denotes the bulk metric components in arbitrary coordinates. In five dimensions, we can write in embedded vielbein $\left\{\mathcal{Z}_{, \mu}^{A}, \eta^{A}\right\}$ for the metric of the bulk in the vicinity of $V_{4}$

$\mathscr{G}_{A B}=\left(\begin{array}{cc}\tilde{g}_{\mu v} & 0 \\ 0 & 1\end{array}\right)$.

The perturbed coordinate $\mathcal{Z}^{A}$ is given by the Lie transport $L$ such as

$\mathcal{Z}_{, \mu}^{A}=\mathscr{X}_{, \mu}^{A}+\delta y L_{\eta \mathscr{X}_{, \mu}^{A}}=\mathscr{X}_{, \mu}^{A}+\delta y \eta_{, \mu}^{A}$,

$\eta^{A}=\eta^{A}+\delta y[\eta, \eta]^{A}=\eta^{A}$.

and the normal vector is $\eta^{A}$ unspoiled by perturbations [54-56].

It is worth noting that the constant curvature $K_{*}$ is either zero (flat bulk) or it can have positive (de Sitter) or negative (anti-de Sitter) constant curvatures. We chose a flat bulk with $K_{*}=0$. The confined tangent components of the energy-momentum tensor for the bulk $\mathscr{T}_{A B}$ are proportional to the energy-momentum tensor: $\alpha_{*} T_{\mu \nu}=8 \pi G_{N} T_{\mu v}$, where $G_{N}$ is the gravitational Newtonian constant. On the other hand, the vector $T_{\mu a}$ and scalar $T_{a b}$ components are confined in the sense that $T_{\mu a}=0$ and $T_{a b}=0$ prohibit any propagation of matter fields to extra-dimension, but the gravitational field.

The field equations can be obtained by direct calculation of the tangent components of Eq.(3). As shown in details in Refs.( [54-57]) one can write the induced field equations in the embedded space-time from a fivedimensional bulk

$$
\begin{aligned}
\tilde{G}_{\mu v}+\tilde{Q}_{\mu v} & =8 \pi G_{N} \tilde{T}_{\mu v}, \\
\tilde{k}_{\mu[v ; \rho]} & =0,
\end{aligned}
$$

where the energy-momentum tensor of the confined perfect fluid is denoted by $\tilde{T}_{\mu \nu}$. The quantity $\tilde{G}_{\mu \nu}$ denotes the four dimensional non-perturbed Einstein tensor and $\tilde{Q}_{\mu v}$ is the deformation tensor. This term is defined by

$\tilde{Q}_{\mu v}=\tilde{g}^{\rho \sigma} \tilde{k}_{\mu \rho} \tilde{k}_{v \sigma}-\tilde{k}_{\mu v} \tilde{h}^{2}-\frac{1}{2}\left(\tilde{K}^{2}-\tilde{h}^{2}\right) \tilde{g}_{\mu v}$,

where we denote $\tilde{h}^{2}=\tilde{h} \cdot \tilde{h}$ by the mean Gaussian curvature and $\tilde{K}^{2}=\tilde{k}^{\mu v} \tilde{k}_{\mu v}$ denotes the Gaussian curvature. It follows that $\tilde{Q}_{\mu v}$ is a conserved quantity in the sense that

$\tilde{Q}_{; v}^{\mu \nu}=0$.

We point out that the trace of the Codazzi equation in Eq.(8) is composed by the extrinsic curvature terms and the appearance of the deformation tensor $\tilde{Q}_{\mu \nu}$ of Eq.(9) are a novelty typically inherent to embedding models that extend the curvature concept of a pseudo-Riemannian geometry. The brackets in Eq.(8) apply the covariant derivatives to the adjoining indices.

To complete the set of dynamical equations, the extrinsic curvature dynamics is completed by the analysis of the spin of a linear massless spin-2 fields in Minkowski space-time. Counting from Eq.(3), one obtains a total of 20 unknowns $\tilde{g}_{\mu v}$ and $\tilde{k}_{\mu v}$, against 15 dynamical equations. Thus, the remaining equations must come from $\tilde{k}_{\mu v}$ which is an independent symmetric rank-2 tensor. In 1954, Suraj Gupta noted that the Fierz-Pauli equation has a remarkable resemblance with the linear approximation of Einstein's equations for the gravitational field, suggesting that such equation could be just the linear approximation of a more general, non-linear equation for a massless spintwo fields. Gupta found that any spin-2 field in Minkowski space-time must satisfy an equation that has the same formal structure as Einstein's equations [70-73]. As a result, a set of Einstein-like system of equations called the Gupta equations that are defined as a Ricci-like equations in a form

$\tilde{\mathscr{F}}_{\mu \nu}=0$,

where they are defined as a copy of the usual Riemannian geometry. Hence, once can define a "f-Riemann tensor"

$$
\tilde{\mathscr{F}}_{\mu \nu \alpha \lambda}=\partial_{\alpha} \Upsilon_{\mu \lambda v}-\partial_{\lambda} \Upsilon_{\mu \alpha v}+\Upsilon_{\alpha \sigma \mu} \Upsilon_{\lambda v}^{\sigma}-\Upsilon_{\lambda \sigma \mu} \Upsilon_{\alpha v}^{\sigma}
$$

constructed from a "connection" associated with $\tilde{k}_{\mu v}$. It is worth noting that this is not a bimetric model likewise Rosen Bimetric theory [74] since the true background geometry of the embedded space-time is defined by the metric tensor $\tilde{g}_{\mu v}$. Thus, we define the tensors

$\tilde{f}_{\mu v}=\frac{2}{\tilde{K}} \tilde{k}_{\mu v}$, and $\tilde{f}^{\mu v}=\frac{2}{\tilde{K}} \tilde{k}^{\mu v}$,

so that the normalization condition $\tilde{f}^{\mu \rho} \tilde{f}_{\rho v}=\delta_{v}^{\mu}$ is applied and $\tilde{K}=\sqrt{\tilde{k}_{\mu v} \tilde{k}^{\mu v}}$. Accordingly, it is possible to 
define the "Levi-Civita connection" associated with $\tilde{f}_{\mu v}$, based on the analogy with the "metricity condition" $\tilde{f}_{\mu v \| \rho}=0$, where the double bars $\|$ denote the covariant derivative with respect to $f_{\mu v}$ (while keeping the usual semicolon sign for the covariant derivative with respect to $\tilde{g}_{\mu v}$ ). The "f-connection" is given by

$$
\Upsilon_{\mu v \sigma}=\frac{1}{2}\left(\partial_{\mu} \tilde{f}_{\sigma v}+\partial_{v} \tilde{f}_{\sigma \mu}-\partial_{\sigma} \tilde{f}_{\mu v}\right)
$$

and also the contraction

$$
\Upsilon_{\mu \nu}^{\lambda}=\tilde{f}^{\lambda \sigma} \Upsilon_{\mu \nu \sigma} .
$$

\subsection{Background FLRW embedded universe}

In order to describe the cosmological background, we use Eqs.(7), (8) and (11), with the usual Friedman-LemaîtreRobertson-Walker (FLRW) in coordinates $(r, \theta, \phi, t)$

$d s^{2}=d t^{2}-a^{2}\left[d r^{2}+f_{\mathcal{K}}^{2}(r)\left(d \theta^{2}+\sin ^{2} \theta d \varphi^{2}\right)\right]$,

where $a=a(t)$ is the scale expansion factor, $f(r)_{\kappa}=\sin r$, $\mathrm{r}, \sinh r$ and $\kappa$ corresponds to spatial curvature $(1,0,-1)$. For the present application, we consider a flat universe.

The stress energy tensor in a non-perturbed comoving fluid with a co-moving velocity $u_{\mu}$ is given by

$\tilde{T}_{\mu v}=(\tilde{\rho}+\tilde{p}) u_{\mu} u_{v}-\tilde{p} \tilde{g}_{\mu v} ; u_{\mu}=\delta_{\mu}^{4}$.

The related conservation equation

$\tilde{\rho}+3 H(\tilde{\rho}+\tilde{p})=0$,

where $\tilde{\rho}$ and $\tilde{p}$ denote non-perturbed matter density and pressure, respectively.

The trace of the Codazzi equations given by Eq.(8) has the general solution

$$
\tilde{k}_{i j}=-\frac{b}{a^{2}} \tilde{g}_{i j}, i, j=1,2,3, \quad \tilde{k}_{44}=-\frac{1}{\dot{a}} \frac{d}{d t} \frac{b}{a},
$$

where the dot symbol denotes time derivative. The bending function $b(t)=k_{11}$ carries the arbitrariness from the confinement of the gauge fields in a five dimensional bulk leading to the homogeneity of the trace of the Codazzi equations as shown in Eq.(8).

Taking the standard cosmological notation, we denote the usual Hubble parameter defined by $H(t)=\dot{a} / a$ and analogously we define the extrinsic cosmic parameter as $B(t)=\dot{b} / b$. Thus, we summarize the results presented in
$[56,57]$ such as

$$
\begin{aligned}
& \tilde{k}_{44}=-\frac{b}{a^{2}}\left(\frac{B}{H}-1\right), \\
& \tilde{K}^{2}=\frac{b^{2}}{a^{4}}\left(\frac{B^{2}}{H^{2}}-2 \frac{B}{H}+4\right), \tilde{h}=\frac{b}{a^{2}}\left(\frac{B}{H}+2\right), \\
& \tilde{Q}_{i j}=\frac{b^{2}}{a^{4}}\left(2 \frac{B}{H}-1\right) \tilde{g}_{i j}, \tilde{Q}_{44}=-\frac{3 b^{2}}{a^{4}}, \\
& \tilde{Q}=-\left(\tilde{K}^{2}-\tilde{h}^{2}\right)=\frac{6 b^{2}}{a^{4}} \frac{B}{H},
\end{aligned}
$$

where in Eq.(18), we have denoted $i, j=1 . .3$ with no sum on indices.

The elimination of the arbitrariness of Eq.(8) is solved by calculating the Gupta equations in Eq.(11) and the conservation equation of the deformation tensor $\tilde{Q}_{; \mu}^{\mu}=0$. Thus, one obtains

$b(t)=\gamma_{0} a^{\beta_{0}} e^{\frac{1}{2} \Gamma(t)}$,

where the $\Gamma$-exponent in the exponential function is defined by

$\Gamma(t)= \pm \sqrt{\left|4 \eta_{0} a^{4}-3\right|} \mp \sqrt{3} \arctan \left(\frac{\sqrt{3}}{3} \sqrt{\left|4 \eta_{0} a^{4}-3\right|}\right)$,

where we adopt the referencing $\Gamma^{(+)}$and $\Gamma^{(-)}$for each solution with respect to the sign \pm . In addition, the $\gamma_{0}$ and $\beta_{0}$ quantities denote integration constants in Eq.(20). The $\eta_{0}$ parameter is originated from the Gupta equations. As cosmography tests indicate [58] the $\eta_{0}$ parameter is related to the magnitude of the width of the transition redshift from a decelerating to accelerating universe and the $\beta_{0}$ parameter affects the magnitude of the deceleration parameter $q=q(z)$ that is given by

$q(z)=\frac{1}{H(z)} \frac{d H(z)}{d z}(1+z)-1$.

Hence, we can write

$q(z)=\frac{3}{2}\left[\frac{\Omega_{m}(z)+\gamma^{*} \Omega_{e x t}(z)}{\Omega_{m}(z)+\Omega_{e x t}(z)}\right]-1$,

where $\gamma^{*}=\frac{1}{3}\left[4-2 \beta_{0} \pm 2 \sqrt{\left|\frac{4 \eta_{0}}{(1+z)^{4}}-3\right|}\right]$. The matter density parameter is denoted by $\Omega_{m}(z)=\Omega_{m}^{0}(1+z)^{3}$ and the term $\Omega_{\text {ext }}(z)=\Omega_{\text {ext }}^{0}(1+z)^{4-2 \beta_{0}} \gamma_{0}$ stands for the density parameter associated with the extrinsic curvature. The upper script " 0 " denotes the present value of any quantity. The relation of the redshift $z$ with the scale expansion factor $a$ is given by $a=\frac{1}{1+z}$. The current extrinsic contribution $\Omega_{\text {ext }}^{0}$ is given by the normalization condition for a 
flat universe with redshift at $z=0$ that results in

$\Omega_{\text {ext }}^{0}=\frac{1}{\gamma_{0} \Gamma(0)}\left(1-\Omega_{m}^{0}\right)$.

In this work, we ignore the radiation contribution since it can be dismissed at late times.

The related Friedmann equations for a flat universe in terms of redshift are written as

$H(z)=H_{0} \sqrt{\Omega_{m}(z)+\Omega_{\text {ext }}(z) e^{\Gamma^{ \pm}(z)}}$,

where $H(z)$ is the Hubble parameter in terms of redshift $z$ and $H_{0}$ is the current value of the Hubble constant. Hence, we can write the dimensionless Hubble parameter $E(z)=$ $\frac{H(z)}{H_{0}}$ as

$E^{2}(z)=\Omega_{m}^{0}(1+z)^{3}+\left(1-\Omega_{m}^{0}\right)(1+z)^{4-2 \beta_{0}} e^{\Gamma^{ \pm}(z)}$.

Since the bending function $b(t)$ has a positive and negative solutions ( $\Gamma^{ \pm}$-solutions), one obtains a family of such solutions for Eq.(26) that depend on the values of $\left(\gamma_{0}, \beta_{0}, \eta_{0}\right)$.

\section{Growth density and numerical results}

\subsection{Scalar perturbations in a conformal Newtonian gauge}

In longitudinal conformal Newtonian gauge, one can write the FLRW metric as

$d s^{2}=a^{2}\left[(1+2 \Phi) d \tau^{2}-\left((1-2 \Psi) \delta_{i j} d x^{i} d x^{j}\right]\right.$,

where $\Phi=\Phi(\mathbf{x}, \tau)$ and $\Psi=\Psi(\mathbf{x}, \tau)$ denotes the Newtonian potential and the Newtonian curvature in conformal time. The expansion parameter in conformal time is denoted as $a=a(\tau)$ from $d t=a(\tau) d \tau$.

We can write the perturbed field equations as

$$
\begin{aligned}
\delta G_{v}^{\mu} & =8 \pi G_{N} \delta T_{v}^{\mu}-\delta Q_{v}^{\mu}, \\
\delta k_{\mu v ; \rho} & =\delta k_{\mu \rho ; v}, \\
\delta F_{v}^{\mu} & =0 .
\end{aligned}
$$

When considering cosmological perturbations, differently from the background framework, we need to understand how the extrinsic curvature evolves. The answer is given by Nash-Greene fluctuations of Eq.(1) to obtain the linear perturbations for five-dimensions for the perturbed extrinsic curvature

$k_{\mu v}=\tilde{k}_{\mu v}-2 \delta y \tilde{g}^{\sigma \rho} \tilde{k}_{\mu \sigma} \tilde{k}_{v \rho}$, where $\delta k_{\mu v}=\tilde{g}^{\sigma \rho} \tilde{k}_{\mu \sigma} \tilde{k}_{v \rho}$. From Eq.(1), we obtain

$\delta k_{\mu v}=\tilde{g}^{\sigma \rho} \tilde{k}_{\mu \sigma} \delta g_{v \rho}$.

Immediately, we obtain the perturbation of the deformation tensor $Q_{\mu v}$ from its background form in Eq.(9) and from Eq.(32) such as

$\delta Q_{\mu \nu}=-\frac{3}{2}\left(\tilde{K}^{2}-\tilde{h}^{2}\right) \delta g_{\mu \nu}$.

A direct consequence of Eq.(32) is that the Codazzi equations in Eq.(29) do not propagate perturbations and are confined to the background. A similar result is obtained to the perturbed Gupta equations in Eq.(30) once we have $\delta f_{\mu v}=0$. Thus, Eqs. (29) and (30) maintain the same background form as in Eqs.(8) and (11), and they not provide additional information to cosmological perturbations in which are only provided by the gravitational tensor equation of Eq.(28). Then using the background relations in Eqs.(16), (17), (18), and (19), we can determine the components of $\delta Q_{\mu v}$

$\delta Q_{j}^{i}=\gamma_{0} a^{2 \beta_{0}-2} \Psi \delta_{j}^{i}$,

$\delta Q_{4}^{i}=0$,

$\delta Q_{4}^{4}=\gamma_{0} a^{2 \beta_{0}-2} \Phi \delta_{4}^{4}$.

For a perturbed fluid with pressure $p$ and density $\rho$, one can write the perturbed components of the related stress-tensor

$\delta T_{4}^{4}=\delta \rho$,

$\delta T_{i}^{4}=\frac{1}{a}\left(\rho_{0}+p_{0}\right) \delta u_{\| i}$,

$\delta T_{j}^{i}=-\delta p \delta_{j}^{i}$,

where $\delta u_{\| i}$ denotes the tangent velocity potential and $\rho_{0}$ and $p_{0}$ denote the non-perturbed components of density and pressure, respectively. Hence, we obtain the perturbed equations in the Fourier $k$-space wave modes in a form

$$
\begin{array}{r}
k^{2} \Psi_{k}+3 \not{H}\left(\Psi_{k}^{\prime}+\Phi_{k} \not{H}\right)=-4 \pi G_{N} a^{2} \delta \rho_{k}+\gamma_{0} a^{2 \alpha_{0}} \Phi_{k},(40) \\
\Psi_{k}^{\prime}+\not{H} \Phi_{k}=-4 \pi G_{N} a^{2}\left(\rho_{0}+p_{0}\right) \theta,(41) \\
\Psi_{k}^{\prime \prime}+\not{H}\left(2 \Psi_{k}+\Phi_{k}\right)^{\prime}+\left(\mathscr{H}^{2}+2 \mathscr{P}^{\prime}\right) \Phi_{k}+\frac{1}{2} k^{2}\left(\Psi_{k}-\Phi_{k}\right)(42) \\
=\frac{1}{2} \hat{k}^{i} \cdot \hat{k}_{i}\left(\Psi_{k}-\Phi_{k}\right)+4 \pi G_{N} a^{2} \delta p+\gamma_{0} a^{2 \alpha_{0}} \Psi_{k} .
\end{array}
$$

where $\theta=i k^{j} \delta u_{\| j}$ denotes the divergence of fluid velocity in $k$-space. 


\subsection{Matter density evolution under sub-horizon regime}

In the following we do not consider any anisotropic stress effect and pressure from the matter contribution in order to analyse the gravitational signal from the contribution of the extrinsic curvature, and we focus on the fluctuations of the Bardeen fields $\Phi$ and $\Psi$. It is important to notice that when $\gamma_{0} \rightarrow 0$ in set of Eqs.(40), (41) and (43), the standard GR equations are obtained. It means we can recover the sub-horizon approximation with $k^{2} \gg \mathscr{\ell}^{2}$ or $k^{2} \gg a^{2} H^{2}$ which means $\Phi_{k}^{\prime \prime}, \mathscr{H} \Phi_{k}^{\prime}, \mathscr{H} \Phi_{k}^{\prime} \sim 0$, then Eq. (40) turns the expected Newtonian formula $\Phi_{k} \sim \frac{\delta \rho_{k}}{k^{2}}$. Thus, we obtain the relevant equations at sub-horizon scales

$$
\begin{array}{r}
k^{2} \Psi_{k}-\gamma_{0} a^{2 \beta_{0}} \Phi_{k}=-4 \pi G_{N} a^{2} \delta \rho_{k}, \\
k^{2}\left(\Psi_{k}-\Phi_{k}\right)=-2 \gamma_{0} a^{2 \beta_{0}} \Psi_{k} .
\end{array}
$$

For the present application, even with the dismissal of the matter anisotropic stress, Eq.(44) shows that the extrinsic curvature contributes with anisotropic effect by the field frictions. In this sense, we define the following anisotropic parameters

$\eta(a)=\frac{\Phi-\Psi}{\Psi}$,

and also the slip

$\gamma(a)=\frac{\Psi}{\Phi}$.

Calculating the components $\delta T_{\mu ; 4}^{\mu}$ and $\delta T_{\mu ; i}^{\mu}$, and performing the definition of the "contrast" matter density $\delta_{m} \equiv \frac{\delta \rho}{\rho_{0}}$ in terms of the expansion factor $a(t)$, we obtain the equation

$$
\begin{array}{r}
\frac{d^{2} \delta_{m}(a)}{d a^{2}}+\left(\frac{3}{a}+\frac{1}{H(a)} \frac{d H(a}{d a}\right) \frac{d \delta_{m}(a)}{d a} \\
-\frac{3}{2} \Omega_{m 0} \frac{G_{e f f} / G}{\left(H^{2}(a) / H_{0}^{2}\right)} \delta_{m}(a)=0, .
\end{array}
$$

which solutions are possible only numerically. For instance, in the context of GR, where $G_{\text {eff }}=G_{N}$ that turns $\delta_{m}(a)$ independent of the scale $k$, in terms of the fluid parameter $w=\frac{p_{0}}{\rho_{0}}$, one has the following solution

$\delta_{m}(a)=a \cdot 2 F_{1}\left(-\frac{1}{3 w}, \frac{1}{2}-\frac{1}{2 w} ; 1-\frac{5}{6 w} ; a^{-3 w}\left(1-\Omega_{m}^{-1}\right)\right)$

where $2 F_{1}(a, b ; c ; z)$ is a hypergeometric function.

And from Eqs.(43) and (44), we obtain the effective field

$\Phi_{k}(k, a)=-\frac{4 \pi G_{N}}{k^{2}} \frac{G_{e f f}}{G_{N}} a^{2} \rho_{m} \delta_{m},$. where $G_{e f f} / G_{N}$ is given by

$$
\frac{G_{e f f}(k, a)}{G_{N}}=\frac{1}{\left(1-\frac{\gamma_{0}}{k^{2}} a^{2 \beta_{0}}\right)}-\frac{2 \gamma_{0} Q_{e f f}(k, a) a^{2 \beta_{0}}}{k^{2}\left(k^{2}-\gamma_{0} a^{2 \beta_{0}}\right)} .
$$

and also

$\Psi_{k}(k, a)=-\frac{4 \pi}{k^{2}} G_{N} Q_{e f f} a^{2} \rho_{m} \delta_{m}$,

where $Q_{e f f}=\frac{1}{1-\frac{\gamma_{0}}{k^{2}} a^{2} \beta_{0}+2 \frac{\gamma_{0}^{2}}{k^{4}} a^{4 \beta_{0}}}$.

The form of the effective Newtonian constant $G_{\text {eff }}$ is given by Eq.(50) as a result from Nash-Green fluctuations of the background. In order to obtain some constraints on the parameters, namely, $\left(\eta_{0}, \gamma_{0}, \beta_{0}\right)$, we analyse numerically the Eq.(50). Firstly, we adopt a minimum value of expansion parameter $a_{\min }=0.001$ to guarantee that the analysis is restricted to the matter domination scale such as for sub-horizon scales we fix the value of $k=300 H_{0} \sim 0.1 \mathrm{hMpc}^{-1}$ which was rescaled in a sense $\tilde{k}=H_{\text {ref }} k$. The tilde symbol refers to the physical $k, H_{\text {ref }}$ denotes the Hubble expansion as a reference and the bare new dimensionless $k$ is set as $k=300 \frac{H_{0}}{H_{\text {ref }}}$. The same criteria was applied to other relevant quantities such as, the Hubble constant $\tilde{H}_{0}=H_{r e f} H_{0}, \tilde{\rho}_{m}=H_{r e f}^{2} \rho_{m}$, $\tilde{k}=k_{r e f} k$, and also the fields $\tilde{\Phi}=\Phi_{r e f} \Phi$ and $\tilde{\Psi}=\Psi_{r e f} \Psi$ with $\frac{\Phi_{r e f}}{\Psi_{r e f}}=1$. The overall form of the related equations are kept intact.

After these considerations, we are able to analyse the evolution of $G_{\text {eff }}$ function. It obeys Big Bang Nucleosynthesis and solar experiment constraints in such a way $\left.\left.G_{e f f}\right\rfloor_{a=0}=1, G_{e f f}\right\rfloor_{a=1}=1$ and by the ordinary timederivative $\left.\frac{d G_{e f f}}{d t}\right\rfloor_{a=1}=0$ [43]. The latter relation is satisfied when the extrinsic curvature vanishes $\gamma_{0} \rightarrow 0$, which means that the extrinsic curvature is closer to zero at solar scale as expected and the bare gravitational Newtonian constant $G_{N}$ is recovered. It is understood that only at scales larger than the Newtonian solar scale the extrinsic curvature imprints more a dynamical influence and may drive topological changes of the universe $[54,56,57,59,60]$.

Since Eq.(25) provides a family of solutions with the parameters $\left(\eta_{0}, \gamma_{0}, \beta_{0}\right)$, we can constrain the model by the fact that the simplest cosmological solution relies on $\Lambda \mathrm{CDM}$ at background level, whereas we naturally expect a rather different situation at perturbation level. The similarity of Hubble function $H(a)$ of $\Lambda$ CDM background is only obtained at first order when expanding the Hubble function around $a=0$ and fixing $\beta=2, \eta_{0}=0$. Hence, it is reasonable that values around $\beta_{0}=2$ induce different results at background level departing from the $\Lambda \mathrm{CDM}$ standards. 


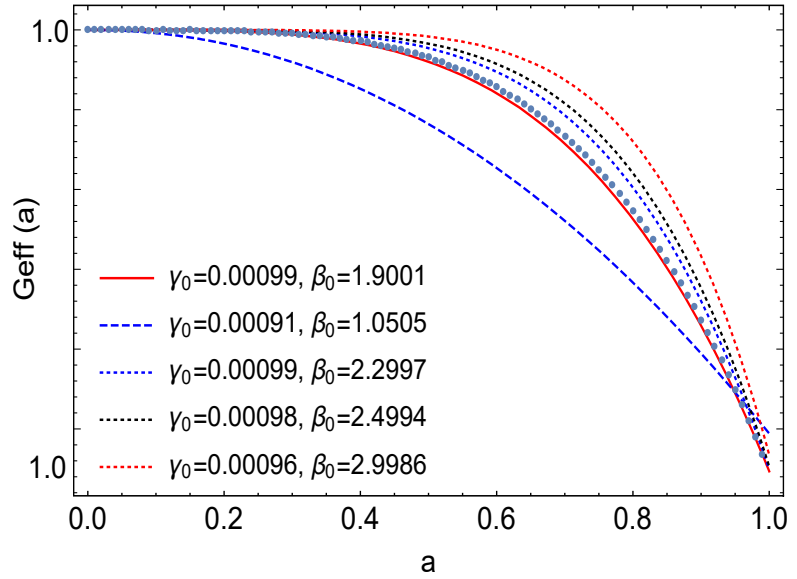

Figure 1 The panel shows the plot of $G_{e f f}$ function with curves defined by numerical values of $\left(\gamma_{0}, \beta_{0}\right)$. The dotted blue line shows datapoints numerically calculated in the range $a=\left[a_{\min }, 1\right]$.

\subsection{Numerical analysis on $G_{\text {eff }}$ and effective Newtonian fields}

We adopt for both cases of $\Gamma^{ \pm}(a)$ the initial values of $\gamma_{0} \rightarrow 0.001$ and $\beta_{0} \rightarrow 2$. The numerical results of the $G_{\text {eff }}$ function is shown in the plot of Fig.(1). The related numerical datapoints were calculated within matter dominated era in the range $a=\left[a_{m i n}, 1\right]$ with the priors on $\left(\gamma_{0}, \beta_{0}\right)$ parameters. We observed that only the curves constructed in the range $\beta_{0}=[1.9,3]$ are well accommodated to datapoints and obey the constraints on $G_{\text {eff }}$ with lowvalues of $\gamma_{0} \leq 0.001$. On the other hand, higher values of $\gamma_{0}>0.001$ and values of $\beta_{0}<1.9$ and $\beta_{0}>3$ gradually break down the $G_{\text {eff }}$ constraints with $G_{\text {eff }}(a=1) \neq 1$ as shown by the dashed blue line in Fig.(1).

From the numerical results of the analysis of Eq.(50) with a constraints on $\left(\eta_{0}, \gamma_{0}, \beta_{0}\right)$ allow us to solve numerically the density growth equation in Eq.(47). To facilitate referencing, we refer to our model as $\beta$-model when necessary. Looking for a well behaved solutions for positive and negative $\Gamma$, we adopt the values $\left(\gamma_{0}=0.001, \beta_{0}=2.3\right)$ for the first model, $\left(\gamma_{0}=0.0001, \beta_{0}=2.5\right)$ for the second one, and $\left(\gamma_{0}=0.0001, \beta_{0}=3\right)$ for the third one. The initial conditions for Eq.(47) are set by $\delta_{m}\left(a_{\min }\right)=a_{\min }$ and the related derivative with respect to expansion factor $\delta_{m}^{\prime}\left(a_{\text {min }}\right)=1$. The results are presented in Fig.(2) with plots of numerical solutions for $\Lambda$ CDM (black line), GR quintessence (blue dashed line) and the $\beta$-model family with the values $\beta_{0}=2.3$ (dotted blue line), $\beta_{0}=2.5$ (dashed magenta line) and $\beta_{0}=3$ (dotted magenta line).
The top right and left panels show the results for the matter growth $\delta_{m}(a)$ for $\Gamma^{(+)}$and $\Gamma^{(-)}$. For $\Gamma^{(+)}$solution, it shows a close compatibility at early times between the models and higher values of matter density growth of the model $\beta_{0}=3$ (dotted magenta line) at late times as compared to $\delta_{m \Lambda C D M}$ (solid black line) and GR quintessence (dashed blue line). For $\Gamma^{(-)}$solution in the top left panel, just as the previous case, it shows a close compatibility at early times between the models and more higher values of matter density for the same set of values of the $\beta$-model at late times as compared to $\delta_{m \Lambda C D M}$ and $\delta_{m}$ for GR quintessence.

In the bottom panels, we can identify more clearly the differences of the rate of the growth by the ratio $\delta_{i} / \delta_{\Lambda C D M}$ with the label $i$ denoting each model in comparison with $\Lambda \mathrm{CDM}$ and GR quintessence. Just as in the previous case (top panels), at early times the models are indistinguishable. On the other hand, at late times they are dramatically different. For both $\Gamma^{( \pm)}$solutions, they depart from $\Lambda \mathrm{CDM}$ pattern around $a=0.2$. As compared with $\Lambda \mathrm{CDM}$, the maximum amplification of overdensities occur with the model for $\beta_{0}=3$ for both $\Gamma$-solutions. It reaches around $6 \%$ for $\Gamma^{(+)}$(bottom right panel) and $14 \%$ for $\Gamma^{(-)}$. In contrast with $\Gamma^{(-)}$solution, the $\Gamma^{(+)}$for $\beta_{0}=2.3$ presents a mild damping of the growth of structures with a transition around $a \sim 0.9$ getting closer to GR quintessence pattern. This is interesting fact since it might be taken into consideration into the dark matter debate on the observed underdensities on local Universe [75]. In summary, $\beta$-model shows that the perturbations from extrinsic curvature provide the necessary gravitational power at least in sub-Hubble scale allowing room for an overall amplification up to $14 \%$ that may leave a trace in the CMB angular power spectrum. Moreover, we analyse the percentage relative difference $\% \operatorname{diff}\left(H-H_{j}\right)$ between the $\beta$-models $H, H 1$ and $H 2$ for the values $\beta_{0}=2.3,2.5,3$, respectively. Specifically, we show the difference with $H_{\Lambda C D M}$ for both $\Gamma$-solutions as shown in Fig.(3). In those cases, we obtained at least a $10 \%$ difference for the lowest value of the model $\beta_{0}=2.3$ and will be larger for higher values of $\beta_{0}$. This corroborates that the $\beta$-model dynamics is rather different in comparison with $\Lambda \mathrm{CDM}$ (or GR quintessence) due to the presence of the $G_{\text {eff }}$ function.

In order to study the evolution of the Newtonian fields in Eqs.(49) and (51) they can be written in a more appropriated form

$\Phi_{k}(k, a)=-\frac{3}{2 k^{2}} \Omega_{0} H_{0}^{2} \frac{G_{e f f}}{G_{N}} a^{2} \rho_{m} \delta_{m},$.

and also

$\Psi_{k}(k, a)=-\frac{3}{2 k^{2}} \Omega_{0} H_{0}^{2} Q_{e f f} a^{2} \rho_{m} \delta_{m},$. 

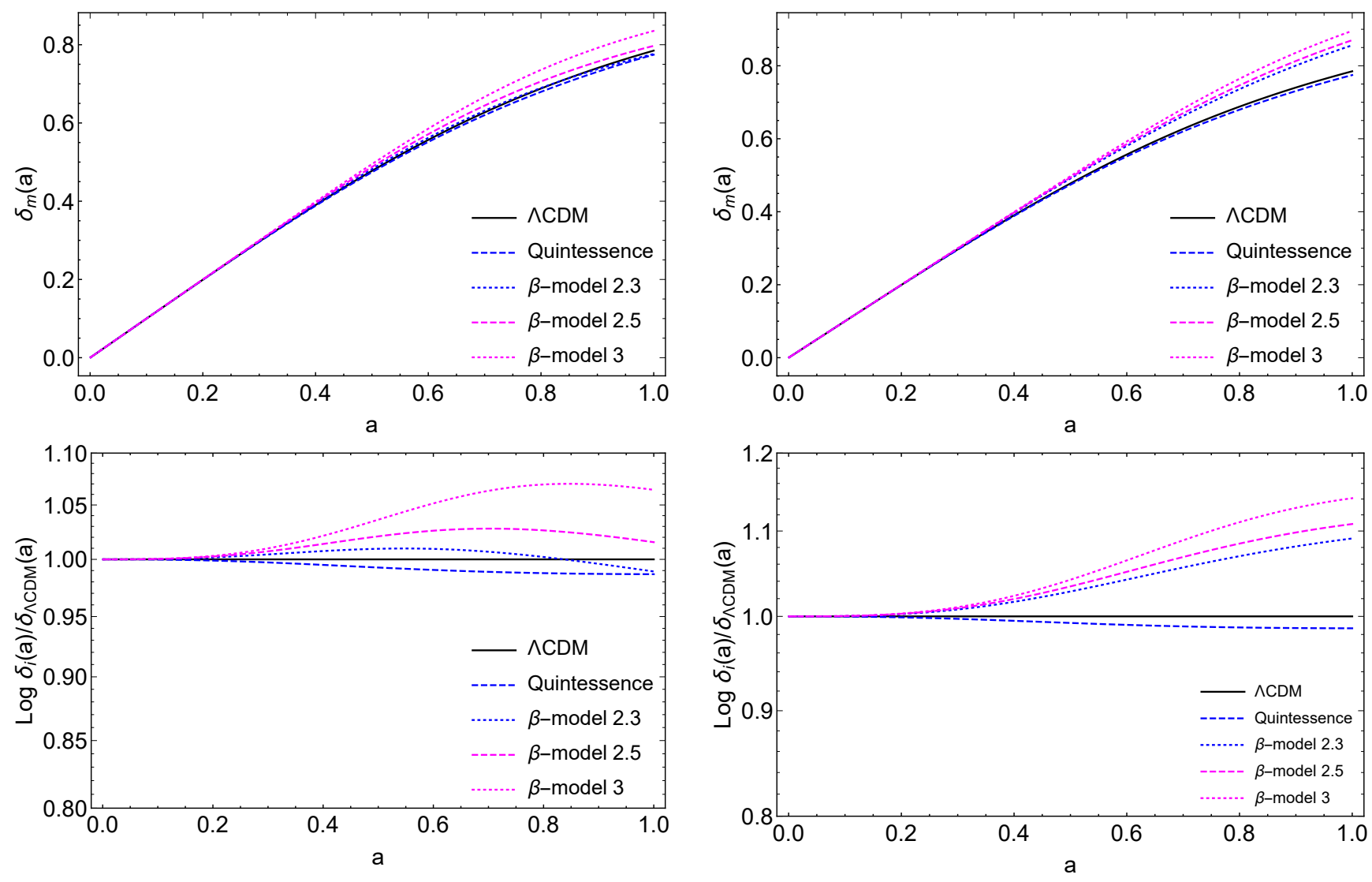

Figure 2 Numerical solution of Matter density perturbations. The top panels show the comparisons of $\Lambda \mathrm{CDM}$, GR quintessence and $\beta$-model values for $\Gamma^{( \pm)}$solutions. The bottom panels show the comparison with these models by the ratio $\delta_{i} / \delta_{m \Lambda C D M}$.
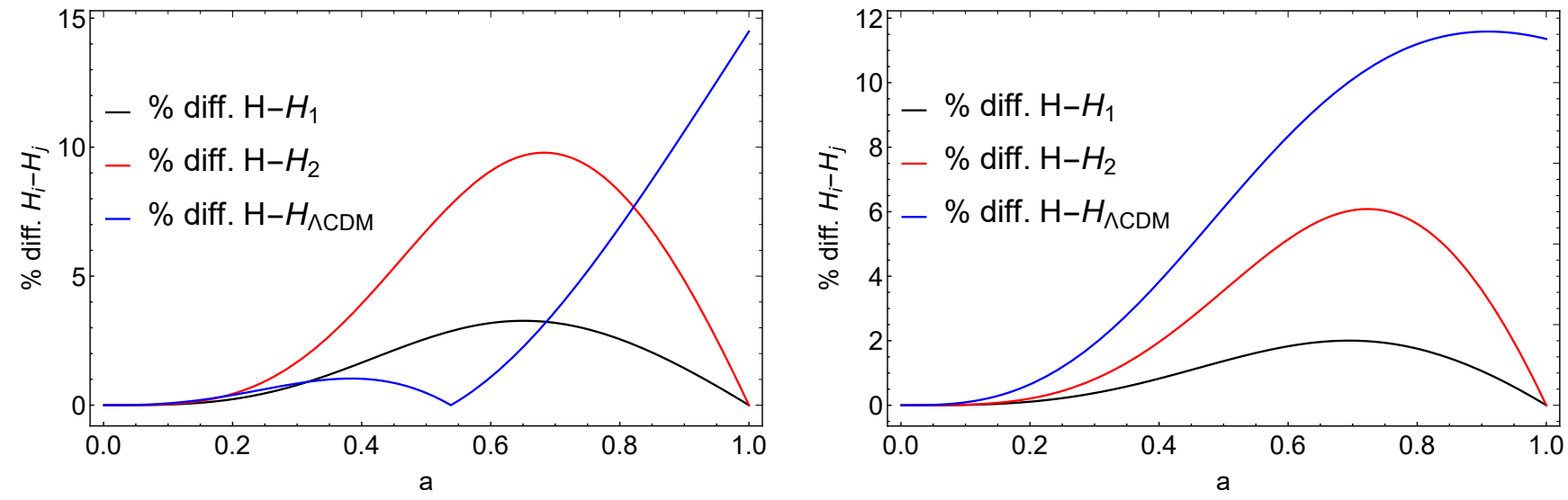

Figure 3 The percentage relative difference $\% \operatorname{diff}\left(H-H_{j}\right)$ between the $\beta$-models for $\Gamma^{( \pm)}$-solutions. The blue line shows a comparison with $H_{\Lambda C D M}$ with a minimum of $10 \%$ percentage difference.

Moreover, we use the conservation equation that of Eq.(15) with the initial value $\rho_{m}\left(a_{m i n}\right)=a_{m i n}^{-3}$ and the

numerical results from $\delta_{m}$ for each study case of $\beta_{0}$ val- 


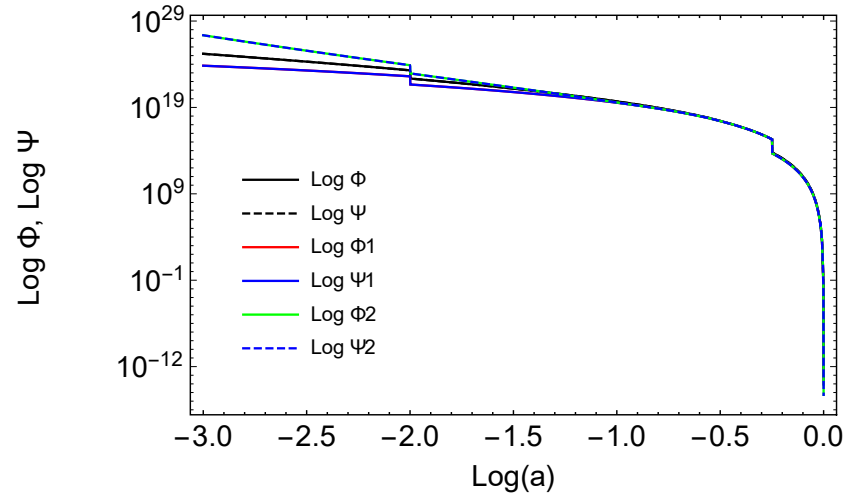

Figure 4 The decaying pattern (Logarithm scale) of the effective Newtonian potentials $\Phi$ and $\Psi$ from early to later times. The $(\Phi, \Psi)$ refers to the $\beta$-model for $\beta_{0}=2.3$. The other models are denoted by $(\Phi 1, \Psi 1)$ for $\beta_{0}=2.5$ and $(\Phi 2, \Psi 2)$ for $\beta_{0}=3$.

ues in different $\Gamma$-solutions. The initial field values are denoted by $\Phi_{i n i}=\Phi_{i n i}\left(a_{m i n}\right)$ and $\Psi_{i n i}=\Psi_{i n i}\left(a_{m i n}\right)$ are calculated at $a_{\min }$. The results of the behaviour of the effective Newtonian potentials $\Phi$ and $\Psi$ are presented in Fig.(4). It is interesting to note that at early times the potentials present higher values that rapidly (but smoothly) decay into lower values with a difference of 40 decimal places in logarithm scale, but are closer to 1 in linear scale calculated at $a=a_{0}=1$. This reinforces the physical robustness of our numerical analysis since the convergence of those potential are expected in Newtonian solar scale. This pattern happens to both $\Gamma$-solutions. The observed "mild steps" in the curves are due to the change of signs of Eqs. (52) and (53) that induce mild fluctuations at that range but not capable of change the form of the curves into amplified oscillations. It may be possible that such amplifications may occur in a larger scale which is different from the dark energy perturbations or modified gravity models such $\mathrm{F}(\mathrm{R})$ and variants, that are mostly negligible at larger scales [76]. In each model, the presented curves of $\Phi$ and $\Psi$ are indistinguishable.

The anisotropy parameters in Eqs. (45) and (46) show better the difference between the fields indicating a physical beyond GR. The $\eta(a)$ parameter measures the relative change between $\Phi$ and $\Psi$, in a meanwhile the $\gamma(a)$ indicates the absolute rate of change between them. In terms of these parameters, the variation of the $\Gamma$-solutions are practically indistinguishable and we present the results in Fig.(5) and Fig.(6) valid for both solutions. In what concerns Fig.(5), higher values of $\beta_{0}$ lead to lower values in early times. Interestingly, lower values of $\beta$ parameter

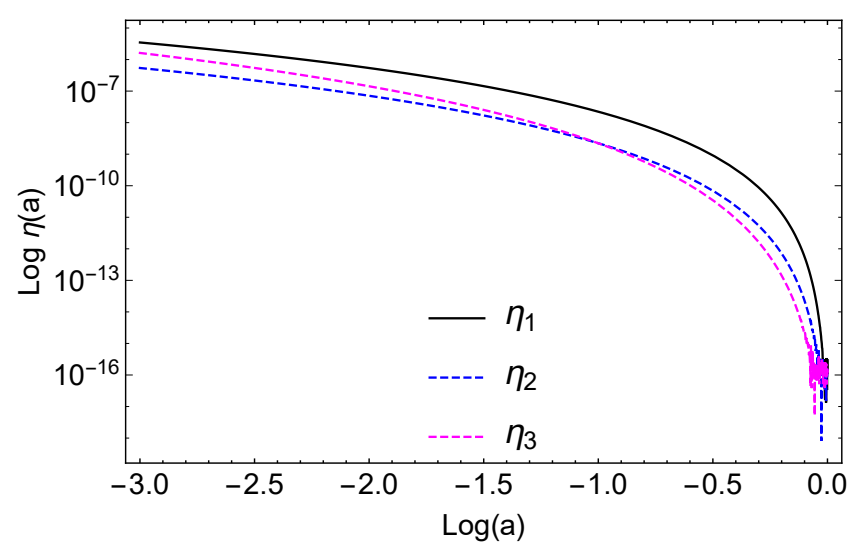

Figure $\mathbf{5}$ The decaying pattern (Logarithm scale) of the anisotropy parameter $\eta(a)$ from early to later times. The bare $(\eta)$ refers to the $\beta$-model for $\beta_{0}=2.3$. The other models are denoted by $(\eta 1)$ for $\beta_{0}=2.5$ and $(\eta 2)$ for $\beta_{0}=3$.

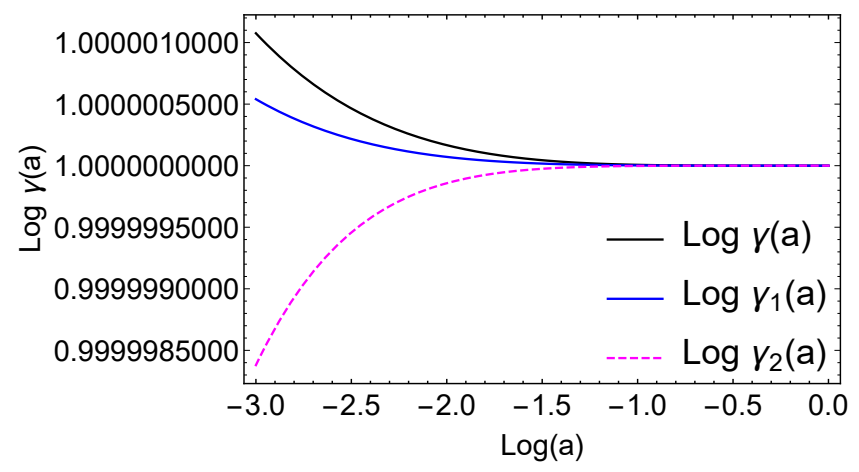

Figure 6 The decaying pattern (Logarithm scale) of the anisotropy parameter $\gamma(a)$ from early to later times. The bare $(\gamma)$ refers to the $\beta$-model for $\beta_{0}=2.3$. The other models are denoted by $\gamma 1$ for $\beta_{0}=2.5$ and $\gamma 2$ for $\beta_{0}=3$.

tends to provoke a higher difference between the potentials at early times. For instance, this is not expected in GR- $\Lambda$ CDM in which the anisotropy parameter $\eta$ is invariantly zero and $\gamma$ is equal 1 for any period. Moreover, all the values converge at late times with mild fluctuations (mild spikes in Fig.(5)), which is corroborate by the anisotropic $\gamma(a)$ showing a mild difference in early times and a convergence for late times. It reinforces our priors that of $\frac{\Phi_{a_{0}}}{\Psi_{a_{0}}}=1$. We stress that the analysis was taken just considering the fluctuations originated by the extrinsic curvature eventually, as we can see in Eq.(44) and a more explicit difference is expected by adding the matter anisotropic stresses. 


\section{Summary}

In this paper, we have analyzed the evolution of matter growth perturbations in the context of an embedding model in five dimensions to complement the concept of curvature in GR with the natural appearance of the extrinsic curvature. Our first results are related to the obtainment of the perturbed equations. Using the linear NashGreen fluctuations, we have shown that only the gravitational tensor equation in Eq.(28) propagates cosmological perturbations, but the trace of Codazzi equation in Eq.(29) and Gupta equations in Eq.(30) remain confined to their background form. In the sub-horizon regime, we obtained a closed form of the effective Newtonian gravitational field $\Phi$ and the effective Newtonian curvature $\Psi$ modified by the presence of extrinsic. The related effective Newtonian function $G_{e f f}$ was calculated numerically to constrain the parameters $\left(\gamma_{0}, \beta_{0}\right)$ of the model since $G_{e f f}$ constrains $\left.G_{e f f}\right\rfloor_{a=0}=1$ and $\left.G_{e f f}\right\rfloor_{a=1}=1$ must be in compliance of both Big Bang Nucleosynthesis and solar constraints. To guarantee a $\Lambda$ CDM background as an initial reference for comparison, the $\eta_{0}$ parameter was fixed to zero and was used to analyze the two $\Gamma$-solutions that appear in the bending function $b(t)$ as shown in Eqs. (20) and (21). It is important to note that the obtained $G_{\text {eff }}$ function does not depend on the $\Gamma$-solution and reinforces its use to constrain the values for the parameters. Moreover, we obtained a tight range for allowed values for $\beta_{0}=[1.9,3]$ and $\gamma_{0}<0.001$. The resulting numerical analysis of growth equations leads to both scenarios of overdensities and a mild damping the growth of structures (for a specific case with $\beta_{0}=2.3$ ) as compared with $\Lambda \mathrm{CDM}$ and GR quintessence. This results pinpoint an interesting direction to discussion of local voids within the dark matter halos debate. It was also shown the relative comparison of the $\beta$-model with GR Lambda in which we obtain a range of $10 \%$ up to $14 \%$ of amplification of the matter fluctuations. This shows an indication of the extrinsic curvature dynamics may provide the necessary gravitational power to explain the growth of matter at large scale and the accelerated regime in the expansion of the universe and may imprint signatures on the CMB power spectrum.

In addition, we also have studied the evolution of the effective Newtonian potentials $\Phi$ and $\Psi$. We have found a robust numerical compatibility between the initial priors and related results that indicate a mild difference of those effective fields at early times and a convergence at late times to a lower values close to 1 that indicate a new physics different from GR. Those mild differences and fluctuations can be amplified with a consideration of the anisotropic stress from matter. Finally, we intend to investigate further the implications of the obtained growth equation within a larger approach considering anisotropic matter stress and the level of sensibility the viscosity parameter within this framework. Also, the integrated Sachs-Wolfe effect to analyse an signature on CMB power spectrum will be a subject of future research.

\section{Acknowledgements}

The author thanks Federal University of Latin-American Integration for financial support from Edital PRPPG 110 (17/09/2018) and Fundação Araucária/PR for the Grant CP15/2017-P\&D 67/2019.

Key words. Embedding; Gravity; Growth matter evolution;Effective Newtonian constant

\section{References}

[1] Y. B. Zeldovich, JETP Lett. 6 316, 1967; Soviet Physics Uspekhi,11 381, 1968.

[2] S. Weinberg, Rev.Mod.Phys., 61, 1, 1989.

[3] S. Weinberg, The Cosmological Constant Problems (Talk given at Dark Matter 2000, February, 2000), ArXiv:astro-ph/0005265 (2000).

[4] I. Shapiro, Class. Quantum Grav., 25, 103001, 2008.

[5] C. P. Burgess, The Cosmological Constant Problem: Why it's hard to get Dark Energy from Micro-physics, ArXiv:1309.4133 [hep-th].

[6] P. J. Steinhardt, Cosmological Challenges for the 21st Century, in Critical Problems in Physics, ed. V. L. Fitch, D. R. Marlow, M. A. E. Dementi, p. 123, 1997.

[7] H. E. S. Velten, R. F. vom Marttens, W. Zimdahl, Eur. Phys. J. C74, 3160, 2014.

[8] S. Perlmutter, et al. Astrophys. J. , 517, 565, 1999.

[9] A. Riess, et al., Astron. J. 116, 1009, 1998.

[10] V. Sahni, A. Starobinsky, Int. J. Mod. Phys. D15, 2105, 2006.

[11] W. J. Percival et al., Mon. Not. Roy. Astron. Soc. 381, 1053, 2007.

[12] S. Alamet et al. (BOSS), Mon. Not. Roy. Astron. Soc. 470, 2617, 2017.

[13] M. Kowalskiet al. (Supernova Cosmology Project), Astrophys. J. 686, 749, 2008.

[14] A. H. Jaffeet al. (Boomerang), Phys. Rev. Lett. 86, 3475, 2001.

[15] L. Izzo, M. Muccino, E. Zaninoni, L. Amati, M. D. Valle, Astron. Astrophys. 582, A115, 2015.

[16] G. Efstathiou and P. Lemos, Mon. Not. Roy. Astron.Soc. 476, 151, 2017.

[17] S. W. Allen et al., Mon. Not. Roy. Astron.Soc. 383, 879, 2008. 
[18] E. Baxteret al., Mon. Not. Roy. Astron. Soc. 461, 4099, 2016.

[19] R. Chávez, et al., Mon. Not. Roy. Astron. Soc. 462, 2431, 2016.

[20] N. Aghanim et al., (Planck Collaboration), Planck 2018 results. VI. Cosmological Parameters, ArXiv:1807.06209.

[21] A. Klypin, A. Kravtsov, O. Valenzuela, F. Prada, Astrophys. J. 522, 1, 89-92., 1999.

[22] B. Moore et al., Astrophys. J. Lett. 524, 1, L19-L22, 1999.

[23] E. V. Karukes, P. Salucci, Mon. Not. R. Astron. Soc. 465, 4703-4722, 2017.

[24] Navarro J.F., Frenk C.S., White S.D.M., Ap.J. 490, 493, 1997.

[25] Salucci P., 2001, Mon. Not. R. Astron. Soc. 344, L1., 2001.

[26] Spekkens K., Giovanelli R., Haynes M.P., Astron. J. 129, 2119-2137, 2005.

[27] Ogiya G., Mori M., Astron. J. 793, 1, 2012.

[28] de Blok, W.J.G., Adv. in Astron., Id.789293, 14, 2010.

[29] A. Del Popolo, M. Le Delliou, Galaxies, 5, 17, 2017.

[30] X. Fan, N. A. Bahcall and R. Cen., Astrophy. J. Lett. 490, 123, 1997.

[31] G. E. Addison, et al. Astrophys. J. 853, 2, 119, 2018.

[32] R. A. Battye, T. Charnock, and A. Moss, Phys. Rev. D91, 10, 103508, 2015.

[33] S. Birrer et al., Mon. Not. Roy. Astron. Soc. 484, 4726, 2019.

[34] I. G. Mccarthy et al., Mon. Not. Roy. Astron. Soc. 476 3, 2999, 2018.

[35] E. J. Copeland, M. Sami, S. Tsujikawa, Int. J. Mod.Phys. D15, 1753, 2006.

[36] J. Frieman, M. Turner, D. Huterer, Ann. Rev. Astron.Astrophys. 46, 385, 2008.

[37] L. Perivolaropoulos, AIP Conf. Proc.848, 698, 2006 [arXiv:astro-ph/0601014].

[38] L. Amendola, D. Polarski, S. Tsujikawa, Phys. Rev. Lett. 98, 131302, 2007.

[39] L. Amendola, R. Gannouji, D. Polarski, S. Tsujikawa, Phys. Rev. D75, 083504, 2007.

[40] S. Nojiri, S. D. Odintsov, Phys. Rev. D74, 086005, 2006.

[41] S. Capozziello, S. Nojiri, S. D. Odintsov, A. Troisi, Phys. Lett. B639, 135, 2006.

[42] S. D. Odintsov, V. K. Oikonomou, L. Sebastiani, Nucl. Phys. B923, 608, 2017.

[43] S. Nesseris, G. Pantazis, L. Perivolaropoulos, Phys. Rev. D 96, 2, $023542,2017$.

[44] L. Kazantzidis, L.Perivolaropoulos, Phys. Rev. D 97, 103503, 2018.

[45] R. Gannouji, L. Kazantzidis, L. Perivolaropoulos, D. Polarski, Phys. Rev. D 98, 104044, 2018.

[46] J. Pérez-Romero, S. Nesseris, Phys. Rev. D97, 023525, 2018.

[47] R. Arjona, W. Cardona, S. Nesseris, Phys. Rev.99, 043516, 2019.

[48] Randall L., Sundrum R., Phys. Rev. Lett.83, 3370, 1999.
[49] Randall L., Sundrum R., Phys. Rev. Lett.83, 4690, 1999.

[50] Dvali G., Gabadadze G., Porrati M., Phys. Lett.B485, 208-214, 2000.

[51] B. Carter, J.-P. Uzan, Nucl. Phys. B606, 45, 2001.

[52] J. Nash, Ann. Maths. 63, 20, 1956.

[53] R. Greene, Memoirs Amer. Math. Soc. 97, 1970.

[54] M. D. Maia, E. M. Monte, Phys. Lett. A297, 2, 9, 2002.

[55] M.D. Maia, N. Silva, M.C.B. Fernandes, JHEP 0704, 047 (2007).

[56] M.D. Maia, E.M. Monte, J.M.F Maia, J.S. Alcaniz, Class. Quant. Grav.22, 1623, 2005.

[57] M.D. Maia, A.J.S. Capistrano, J.S. Alcaniz, E.M. Monte, Gen. Rel. Grav.10, 2685, 2011.

[58] A.J.S. Capistrano, Mon.Not.Roy.Astron.Soc.448, 1232, 2015.

[59] A.J.S. Capistrano, L.A. Cabral, Physica Scripta91, 105001, 2016.

[60] A. J. S. Capistrano, L. A. Cabral, Class. Quantum Grav. 33, 245006, 2016.

[61] A. J. S. Capistrano, Ann. Phys. (Berlin), 1700232, 2017.

[62] A.J. S. Capistrano, Phys. Rev. D 100, 064049-1, 2019.

[63] T. Shiromizu, K. Maeda, M. Sasaki, Phys. Rev. D62, 024012, 2000.

[64] E. Anderson, R. Tavakol, Class.Quant.Grav.20, L267, 2003.

[65] V. Sahni, Y. Shtanov, JCAP(2003)0311, 014, 2003.

[66] S. Tsujikawa, M. Sami, R. Maartens, Phys. Rev. D70, 063525, 2004.

[67] Y. Gong, C. Duan, Mon.Not.Roy.Astron.Soc.352, 847, 2004.

[68] R. A. Battye, B. Carter, Phys. Lett. B509, 331, 2001.

[69] N. Arkani-Hamed, S. Dimopoulos, G. Dvali, Phys. Lett. B429, 263, 1998.

[70] W. Pauli, M. Fierz. Proc.R.Soc.Lond. A173,211, 1939.

[71] S.N. Gupta, Phys. Rev.96, 6, 1954.

[72] C. J. Isham, A. Salam, J. Strathdee. Phys. Rev.3, 4, 1971.

[73] C. Fronsdal, Phys. Rev. D18, 3624, 1978.

[74] N. Rosen, Gen. Rev. Grav. 4435, 1973.

[75] H. Böhringer, G. Chon, C. A. Collins, AA 633, A19, 2020.

[76] J. C. Bueno Sanchez, L. Perivolaropoulos, Phys.Rev.D81, 103505, 2010. 